\title{
Unruptured Left Coronary Sinus of Valsalva Aneurysm Causing Right Ventricular Outflow Tract Obstruction Following Coronary Re-Implantation Surgery: Case Report
}

\author{
Azene Dessie Mengistu ${ }^{1,4^{*}}, \mathrm{C} \mathrm{Reddy}^{2}, \mathrm{PV}_{\text {Suresh }}{ }^{1}, \operatorname{Ravi}_{\text {Ramamurthy }}{ }^{1}$, Sunita Maheshwari $^{1}$, \\ Vimal Raj $^{3}$ \\ ${ }^{I}$ Department of Pediatric Cardiology, Narayana Institute of Cardiac sciences (NIHS), Bangalore, \\ Karnataka, India \\ ${ }^{2}$ Department of Pediatric Cardiac Surgery, NIHS, Bangalore, Karnataka, India \\ ${ }^{3}$ Department of Radiology, Mazumdar Shaw Medical Center, Narayana Health City, Bangalore,
}

Karnataka, India

${ }^{4}$ Cardiac-Center Ethiopia, Addis Ababa University, Ethiopia

\begin{abstract}
*Corresponding Author: Azene Dessie Mengistu, Department of Pediatric Cardiology, Narayana Institute of Cardiac sciences(NIHS), Bangalore, Karnataka, India and Cardiac-Center Ethiopia, Addis Ababa University, Ethiopia, Email: admengistu2000@yahoo.com
\end{abstract}

\begin{abstract}
Background: Sinus of Valsalva aneurysms are rare cardiac anomalies which can be congenital or acquired. Unruptured forms of acquired aneurysms are exceedingly rare and are usually associated with conditions that comprise the elastic connective tissue at the junction of the aortic media and the annulus.
\end{abstract}

Case Presentation: We report a case of unruptured left sinus of Valsalva aneurysm with right ventricular outflow tract obstruction following coronary reimplantation surgery for anomalous left coronary artery from the pulmonary artery (ALCAPA) with a brief review of literature.

Conclusion: Sinus of Valsalva aneurysm with right ventricular outflow tract obstruction could be one of the complications of coronary reimplantation surgery done for ALCAPA. Hence, vigilant monitoring by serial echocardiography is suggested in the routine follow up of such patients.

Keywords: Coronary re-implantation surgery, ALCAPA, Sinus of Valsalva aneurysm, right ventricular outflow tract obstruction

\section{INTRODUCTION}

Aneurysms of the sinuses of Valsalva are rare cardiac anomalies which may be acquired or congenital. The congenital aneurysm is more common than the acquired form accounting for $1 \%$ of the congenital anomalies of the heart and circulation $(1,2)$. Ninety percent to $95 \%$ originate in the right coronary sinus and project into the right ventricle or right atrium, and less than $5 \%$ originate in the left coronary sinus(2).

The fundamental histologic defect responsible for a coronary sinus aneurysm is discontinuity of the elastic layer in the aortic media at the junction between the ascending aorta and aortic valve annulus. These aneurysms do not always rupture but may cause symptoms due to a mass effect on adjacent cardiac structures causing right ventricular outflow tract obstruction; compressing the left coronary artery and causing myocardial infarction or causing conduction disturbances including complete heart block by compressing the conduction system $(1,4)$.

Acquired sinus of Valsalva aneurysms are often rare and are most commonly caused by infectious conditions such as bacterial endocarditis, syphilis, tuberculosis and fungal infections (4).They may also be acquired following cardiac surgical interventions after aortic valve replacement or removal of aortic valve calcification $(4,5)$.We report an acquired form of unruptured sinus of Valsalva aneurysm from the left coronary sinus following coronary 
reimplantation surgery for anomalous origin of the left coronary artery from the pulmonary artery(ALCAPA).

\section{Case Presentation}

We report a one year and five month old male child who had undergone ALCAPA repair (left coronary artery (LCA) translocation and reimplantation) at the age of one month from another hospital and referred to our institution for further management. The patient was a product of non consanguineous marriage and was born after uneventful pregnancy by normal delivery with birth weight of $2.25 \mathrm{~kg}$. There was no family history of cardiac problems or sudden death.

After the surgical intervention for the ALCAPA, the child was discharged after uneventful postoperative course in a stable condition. There was no history of hospital admission after discharge. During the second postoperative follow up visit, five months after the ALCAPA repair, there was a suspicion of pseudo aneurysm of aorta during echocardiographic evaluation. However, the reimplanted left coronary artery caliber was normal. When the child was referred to our institution, he was a comfortable playful boy. He had no dysmorphic features or cyanosis. Vital signs were heart rate of $124 / \mathrm{min}$; respiratory rate of $26 / \mathrm{min}$ and oxygen saturation of $96 \%$ in room air. Anthropometric indices were a weight of $11 \mathrm{~kg}$ and height of $90 \mathrm{cms}$.Precordial examination showed grade 3/6systolic ejection murmur at left upper sternal border. The rest of physical examination findings were unremarkable.

Routine hematologic tests were normal except for evidence of mild anemia. Electrocardiogram showed normal sinus rhythm with no ST segment/T wave abnormalities. Twodimensional and Doppler echocardiography revealed an ascending aorta measuring $14 \mathrm{~mm}$ and unruptured aneurysm of left coronary sinus measuring $13 \times 14 \mathrm{~cm}$ at the level of sinus of Valsalva protruding into right ventricular outflow tract (RVOT) with peak pressure gradient (PPG) of $75 \mathrm{mmHg}$ across the RVOT. Good flow in left main coronary artery (LMCA) and good ventricular function with no regional wall motion abnormalities were noted.

Cardiac magnetic resonance imaging (MRI) demonstrated focal wide-necked aneurysm of left coronary sinus. The LMCA abuts the aneurysm and arose just inferior to it (Fig.1).

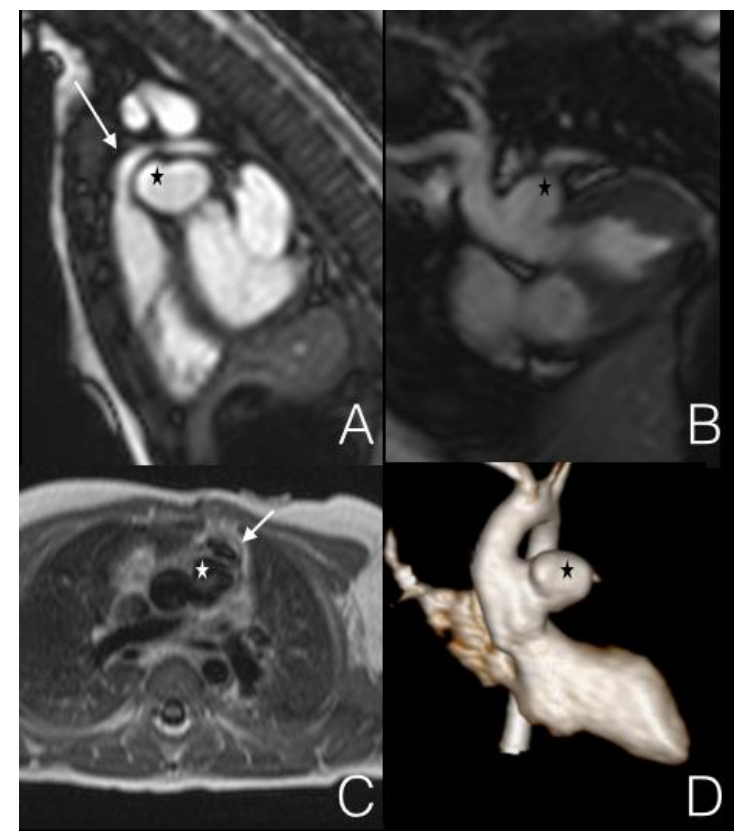

Fig1. Right Ventricle Outflow Tract (RVOT) (A) and axial $(C)$ images showing the aneurysm from the aorta (star) compressing over the proximal pulmonary artery. Left ventricle Outflow Tract (LVOT) (B) and Volume Rendered 3-D (D) image showing the aneurysm (star) from the left coronary cusp of the aorta

Secondary compression of the right ventricular outflow tract was noticed with proximal pulmonary artery circumferential narrowing with flow acceleration in keeping with stenosis (Fig.2).

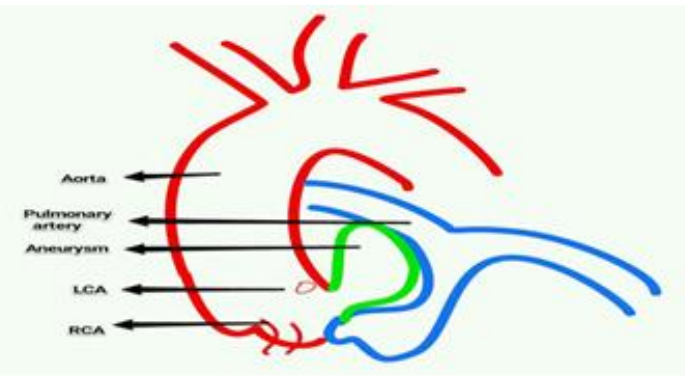

Fig2. Diagrammatic representation of sinus of Valsalva aneurysm from the left sinus causing right ventricular outflow tract obstruction

After the patient was referred for surgical repair the intraoperative findings confirmed the diagnosis and surgical repair of the left sinus of Valsalva aneurysm and relief of RVOT obstruction was performed. Pericardial patch closure of the neck of the aneurysm was done and the aneurysmal sack was marsupialised. The child was discharged after an uneventful postoperative course.

\section{DISCUSSION}

Although rare in occurrence, both ruptured and nonruptured sinus of Valsalva aneurysms are 
associated with potentially fatal complications. These aneurysms may be congenital or acquired. In our case, the fact that the aneurysm originated from the left sinus of Valsalva and the antecedent history of coronary reimplantation surgery favors the diagnosis of an acquired sinus of Valsalva aneurysm. There are few reports in the literature that described acquired sinus of Valsalva aneurysm following a prior cardiac surgery $(1,4,5)$. However, in the English language medical literature we reviewed, there had been no reports of left sinus of Valsalva aneurysm following coronary reimplantation surgery for ALCAPA, suggesting that it is a rare clinical entity.

Unruptured coronary sinus aneurysms are less frequently reported because in most instances, they are clinically silent lesions. As a result of this, most of the literature appears to be biased in favor of ruptured sinus of Valsalva aneurysms. The clinical manifestations of these aneurysms are related to their intrinsic mass effect on the adjacent cardiac structures. The clinical consequences of the mass effect in the nearby structures may be in the form of right ventricular outflow tract obstruction, heart block and coronary artery occlusion resulting in myocardial ischemia (4, 6-11).In adults, unruptured sinus of Valsalva aneurysms may present with chest pain and dyspnea on exertion $(8,9)$. Interestingly, Sundraum, et al from southern India, reported unruptured sinus of Valsalva aneurysm in a patient with inflammatory aortitis (12).

Our case was asymptomatic and thriving well after the surgical repair for ALCAPA. The diagnosis of aneurysm of the sinus of Valsalva with RVOT obstruction was suspected only during a routine follow up echocardiography for the previous ALCAPA repair. There are multiple reports from the adult patient population describing aneurysm of sinus of Valsalva causing RVOT obstruction (8, 9, 11).As our case exemplifies, RVOT obstruction may be one of the manifestations of unruptured sinus of Valsalva aneurysm.

The diagnosis of sinus of Valsalva aneurysm could be established using two-dimensional and Doppler echocardiography. However, both electrocardiographically gated computed tomography and magnetic resonance imaging (MRI) can provide valuable anatomical and functional information (3, 5).In our patient, cardiac MRI was instrumental to establish the diagnosis and functional details prior to the decision for surgical intervention. The mainstay of treatment for ruptured sinus of Valsalva aneurysm is surgical, since patients typically die within one year if the lesion remains uncorrected (10). However, for unruptured aneurysms, specific indications of surgical repair include arrhythmia, right ventricular outflow tract obstruction and large aneurysms $(4,10)$

Translocation of the left coronary artery into the aorta is the optimal operation for ALCAPA when the anatomy is favorable (10). This surgical approach has been implemented since the 1970s with favorable outcomes with no reports of sinus of Valsalva aneurysm in the long term follow up (13-17).

\section{Conclusions}

Sinus of Valsalva aneurysm with right ventricular outflow tract obstruction could be one of the complications of coronary reimplantation surgery done for ALCAPA. Hence, vigilant monitoring by serial echocardiography is suggested in the routine follow up of patients who had undergone such surgical intervention.

\section{CONSENT FOR PUBLICATION}

Written informed consent was obtained from the patient's parents for publication of this Case report and any accompanying images. A copy of the written consent is available for review by the Editor-in-Chief of this journal.

\section{REFERENCES}

[1] Goldberg N., KrasnowN.Sinus of Valsalva Aneurysms.Clin. Cardiol. 1990;13: 831-836

[2] PerloffJK, MarelliAJ. Perloff's Clinical Recognition of Congenital Heart Disease, $6^{\text {th }}$ Edition Elservier Saunders, Philadelphia, 2012; 417-428

[3] Bricker AO, AvutuB, MohammedTH,etal. Valsalva Sinus Aneurysms: Findings at CT and MR Imaging. Radio Graphics 2010; 30:99-110

[4] Weinreich M, Yu P, TrostB.Sinus of Valsalva Aneurysms: Review of the Literature and an Update on Management. Clin. Cardiol. 2015; 38(3): 185-189

[5] Güler N, Eryonucu B, Tuncer M, AşkerM. Aneurysm of sinus of Valsalva dissecting into interventricular septum: a late complication of aortic valve replacement. Echocardiography. 2004; 21(7): 645-8.

[6] KiefaberRW, TabakinBS, Coffin LH, et al. Unruptured Sinus of Valsalva Aneurysm With 
Right Ventricular Outflow Obstruction Diagnosed by Two-Dimensional and Doppler Echo cardiography. J Am Coll CardioI 1986; 7:438-42

[7] Lijoi A, Parodi E, PasseroneGE,etal. Unruptured Aneurysm of the Left Sinus of Valsalva Causing Coronary Insufficiency Case Report and Review of the Literature. Tex Heart Inst J 2002; 29:40-4.

[8] Chung E,BaekJY, Chung HH, et al.A case of unruptured aneurysm of the right sinus of Valsalva with right ventricular outflow tract obstruction. Korean Cir J 2014;(4):274-277

[9] Thankachen R, Gnanamuthu R, DoshiH, etal. Unruptured Aneurysm of the Sinus of Valsalva Presenting with Right Ventricular Outflow Obstruction. Tex Heart Inst J 2003;30:152-4

[10] Kirklin JW, Barratt-Boyes BG. Congenital aneurysm of the sinus of Valsalva. In: Kirklin JW, Barratt-Boyes BG, eds. Cardiac surgery: morphology, diagnostic criteria, natural history, techniques, results and indications, $4^{\text {th }}$ ed.Elsevier Saunders,Philadelphia,2013;1326-1337

[11] FarandP,Brochu M-C,GeravisA, MuellerX. Familial unruptured sinus of Valsalva aneurysm obstructing the right ventricular outflow tract. Can J Cardiol 2009;25(4):227-228
[12] SundaramPS, NarayanGS, PrabhuMA,etal. An unusual cause for unruptured sinus of Valsalva aneurysm. Indian Heart J 2013 ;65: 484-487

[13] Perry LW, Scott LP.Anomalous Left Coronary Artery from Pulmonary Artery Report of 11 Cases; Review of Indications for and Results of Surgery. Circulation. 1970;41:1043-1052

[14] FuduluDP, Dorobantu DM, Sharabiani MTA,etal. Outcomes following repair of anomalous coronary artery from the pulmonary artery in infants: results from a procedure-based national database. Open Heart 2015; 2:e000277. doi:10.1136/openhrt-15-000277

[15] Theodore S,PathakS, Manohar SMK,etal. Anomalous left coronary from the pulmonary artery-Clinical profile, operative techniques and patient outcomes. Ind $\mathbf{J}$ Thorac Cardiovasc Surg, 2005; 21: 148-152

[16] Ramı'rez S, Curi-Curi PJ, Caldero' nColmeneroJ,etal. Outcomes of Coronary Reimplantation for Correction of Anomalous Origin of Left Coronary Artery From Pulmonary Artery. Rev EspCardiol. 2011; 64 (8):681-687.

[17] Muzaffar T, Ganie FH, Swamy SP, WaniN. The Surgical Outcome of Anomalous Origin of the Left Coronary Artery from the Pulmonary Artery. IntCardiovasc Res J.2014;8(2):57-60

Citation: Azene Dessie Mengistu, C Reddy, PV Suresh, Ravi Ramamurthy, Sunita Maheshwari, Vimal Raj. Unruptured Left Coronary Sinus of Valsalva Aneurysm Causing Right Ventricular Outflow Tract Obstruction Following Coronary Re-Implantation Surgery: Case Report. ARC Journal of Clinical Case Reports. 2017, 3(3):20-23. doi:dx.doi.org/10.20431/2455-9806.0303005.

Copyright: (C) 2017 Authors. This is an open-access article distributed under the terms of the Creative Commons Attribution License, which permits unrestricted use, distribution, and reproduction in any medium, provided the original author and source are credited. 